\title{
QUASI RISK-NEUTRAL PRICING IN INSURANCE
}

\author{
BY
}

Harry Niederau and Peter Zweifel

\begin{abstract}
This contribution shows that for certain classes of insurance risks, pricing can be based on expected values under a probability measure $\mathbb{P}^{*}$ amounting to quasi risk-neutral pricing. This probability measure is unique and optimal in the sense of minimizing the relative entropy with respect to the actuarial probability measure $\mathbb{P}$, which is a common approach in the case of incomplete markets. After expounding the key elements of this theory, an application to a set of industrial property risks is developed, assuming that the severity of losses can be modeled by "Swiss Re Exposure Curves", as discussed by Bernegger (1997). These curves belong to a parametric family of distribution functions commonly used by pricing actuaries. The quasi risk-neutral pricing approach not only yields risk exposure specific premiums but also Risk Adjusted Capital (RAC) values on the very same level of granularity. By way of contrast, the conventional determination of RAC is typically considered on a portfolio level only.
\end{abstract}

\section{KEYWORDS}

Incomplete markets, insurance prices, Lorenz order, property risks, quasi riskneutral pricing, risk aversion, risk neutrality, risk management, Risk Adjusted Capital (RAC).

\section{INTRODUCTION}

The theory of pricing risk in incomplete markets based on risk-neutral valuation is well established [see e.g. Delbaen, Haezendonck, 1989, or Gerber, Shiu, 1994]. While a risk-neutral probability measure is not unique in an incomplete market, various approaches have been proposed and discussed for achieving minimum distance of the risk-neutral probability measure with respect to the actuarial measure [see e.g. Föllmer, Schweizer, 1991].

Coherent risk measures, such as expected shortfall [see Artzner, Delbaen, et al., 1999], are widely used in modern Risk Management to measure (aggregated) portfolio risk. At the same time ad hoc pricing methods continue to be 
very much in use by pricing actuaries to price underlying individual risks. This is surprising since distortion principles provide a pricing approach that while applicable to individual risks is consistent with coherent risk measures [see e.g. Dhaene, Vanduffel, et al., 2006]. With respect to assets, Wang (2000) bridged the gap between theory and practice by showing that the Black-Scholes formula can be reproduced by a distortion principle relating to Choquet pricing [see e.g. Denneberg, 1994], introducing the so-called 'Wang transform' to the literature. However, according to Pelsser (2008), the 'Wang transform' does not amount to arbitrage-free pricing independently of the underlying stochastic process.

The focus of this work is to motivate and apply Choquet pricing to industrial property insurance, which by the infrequent nature of loss events (excess of some basic threshold) is typically exposed to heavy tails. Although distortion principles do take into account the extra loading required for heavy-tailed loss distributions, even pricing actuaries familiar with Choquet pricing are hesitant to use them. One reason may be the considerable choice of distortion operators [see e.g. Denuit, Dhaene, et al., 2005, for an overview]. Once a distortion operator has been selected, the task of calibrating in a least arbitrary (and most sensible) way must still be solved.

In the present work we try to overcome these obstacles by proposing both

1. a distortion operator that is optimal in the above stated sense;

2. a unique rule of calibration based on Lorenz order [for a general overview see Denuit, Dhaene, et al., 2005].

A set of insurable risky prospects $X \subset(\Omega, \mathcal{A}, \mathbb{P})$ and a pricing functional,

$$
\begin{aligned}
H:(\Omega, \mathcal{A}, \mathbb{P}) & \rightarrow \mathbb{R}^{+} \\
X & \mapsto H(X),
\end{aligned}
$$

are assumed. Expected values under the actuarial probability measure $\mathbb{P}$ are referred to as actuarial expectation or actuarially fair premium, implying a pricing rule in accordance with the concept of a long-term pure risk premium. We abstract from insurance cycles or price shocks caused by temporary shortages of insurance capacity. The pricing concept presented in this work takes into account the pure loss dynamics underlying an insurance portfolio, risk aversion of market participants and the insurer's solvency. However, it neglects administrative and other expenses, which are very much company-specific.

This paper is structured as follows. Section 2 presents the axiomatic framework that provides the basis for the results reviewed in section 3 and the practical application to follow in section 4 . After applying the theory of quasi risk-neutral pricing to the Swiss Re exposure curves in section 4.1, premiums and Risk Adjusted Capital values are calculated for a property insurance portfolio in section 4.2. Discussion and conclusions with an outlook to further research are given in the final sections 4.3 and 4.4. Appendix A offers some more detail of the pricing rule developed with respect to Swiss Re Exposure 
curves. In turn, Appendix B substantiates the optimality of the exponential distortion principle in section 3 under the criterion of relative entropy minimization, subject to the assumptions made in section 2 .

\section{AXIOMATIC FRAMEWORK AND ESTABLISHED RESULTS}

For ease of reference, we briefly revisit the set of axioms to be satisfied by $H$. With very little variation only these axioms have been extensively discussed in literature [see e.g. Goovaerts, Dhaene, 1998].

Axiom 1 [No Rip-off]. For any risky prospect $X \in X$, we require the pricing functional $H$ to satisfy

$$
\mathbb{E}(X) \leq H(X) \leq \sup (X),
$$

where

$$
\sup (X):=\sup \{X(\omega) \mid \omega \in \Omega\}
$$

If $H(X)=\infty$, a risk is assumed non-insurable.

Axiom 2 [Monotonicity]. The pricing functional $H$ is required to preserve firstorder stochastic dominance in that for any two risky prospects $X_{1}$ and $X_{2}$, such that $X_{1} \leq_{s t} X_{2}$,

$$
X_{1} \leq_{s t} X_{2} \Longrightarrow H\left(X_{1}\right) \leq H\left(X_{2}\right) .
$$

The notation " $X_{1} \leq_{s t} X_{2}$ " means that $X_{2}$ exhibits first stochastic dominance over $X_{1}$ which is defined in terms of distribution functions by the relation

$$
F_{X_{1}}(x) \geq F_{X_{2}}(x), \forall x \in \mathbb{R}^{+} .
$$

It is noted that this monotonicity assumption is weaker than the point-wise notion as discussed in Wang's papers, since it just relates to distribution functions rather than to an underlying probability space. However, on any given probability space point-wise monotonicity implies monotonicity with respect to first stochastic dominance so that this axiom is neither unrelated nor in contradiction to Wang's monotonicity axiom.

Axiom 3 [Subadditivity]. For any two risky prospects $X_{1}$ and $X_{2}$ it must hold true that

$$
H\left(X_{1}+X_{2}\right) \leq H\left(X_{1}\right)+H\left(X_{2}\right)
$$


For a potential purchaser of insurance strictly subadditive premiums, the case of strict inequality in (3), constitute a motivation for transferring risks in particular rather than resorting to self-insurance. However, from the insurer's point of view, subadditive pricing is not an obvious general rule. In terms of the covariance principle for instance, any sort of risk accumulation (e.g. in the context of natural perils or terror risk) may call for super-additive premiums, since the required return on risk capital for risk accumulation zones is higher than otherwise. On the other hand, in a competitive insurance market, insureds would always split their risk between two or more insurers who are well diversified with respect to a potentially accumulating risk. In essence, this argument underlies the concept of 'securitization', i.e. transferring suitably sized and collateralized portions of accumulation risk (e.g. Earthquake California or Earthquake Tokyo) to the capital market. Thus, the subadditivity axiom rules out the possibility of insurers loading their premiums with extra charges for nondiversifying risks.

Axiom 4 [Commonotonic Additivity]. For any two comonotonic risky prospects $X_{1}$ and $X_{2}$,

$$
H\left(X_{1}+X_{2}\right)=H\left(X_{1}\right)+H\left(X_{2}\right)
$$

Comonotonic risks exhibit perfect positive stochastic dependence. For details concerning the concept of comonotonicity, see for instance Denuit, Dhaene, et al. (2002a,b).

Axiom 5 [Continuity]. For any increasing sequence $X_{n}(\omega)>X(\omega), \forall \omega \in \Omega$,

$$
\lim _{n \rightarrow \infty} H\left(X_{n}\right)=H(X) .
$$

For later reference, we recall the representation of $H$ as integral. For some risk $X \in X$ with a continuous distribution function on $\mathbb{R}^{+}$

$$
H(X)=\int_{\mathbb{R}^{+}} x \pi^{\prime}\left(F_{X}(x)\right) f_{X}(x) d x=\int_{\mathbb{R}^{+}} x d\left[\pi\left(F_{X}(x)\right)\right],
$$

where the distortion function $\pi$ ( $\pi^{\prime}$ denotes its derivative) mirrors the above axioms by the following properties,

$(\pi 1) \pi$ is increasing on $(0,1)$;

$(\pi 2) \pi$ is convex on $(0,1)$;

( $\pi 3) \pi[0]=0$ and $\pi[1]=1$. 
Details showing how the above Axioms 1-5 translate into these properties can for instance be found in Goovaerts, Dhaene (1998). An additional property advocated by Wang (2000),

$$
(\pi 4) \pi^{\prime}[1]=\infty
$$

deserves mentioning. It is of practical concern because it counterbalances the decreasing likelihood of large losses, preventing expected loss levels in high layers from approaching zero. Also, as shown in Wang (2000), there is a distortion operator having property $(\pi 4)$ which reproduces the Black-Scholes formula for option pricing. However, this property is not a logical consequence of the axiomatic framework introduced above. Hence, it will not be considered any further in this paper. As a means of emphasis we remind the reader of the above mentioned limitations otherwise as given by Pelsser (2008).

Various additional properties of the operator $H$ can be shown from the above axiomatic framework [see for instance Goovaerts, Dhaene, 1998]. Linearity of the operator $H$ for comonotonic risks and its convexity property are derived explicitly in what follows. Both properties are important for ensuing argument with respect to scaling risk [see Remark 3, section 3] in particular but also in regard of quota-share and excess of loss insurance covers [see section 4.3], respectively.

Proposition 1. For any comonotonic risks $X_{1}$ and $X_{2}$ and arbitrary non-negative scalars $\alpha$ and $\beta$ it holds true that

$$
H\left(\alpha X_{1}+\beta X_{2}\right)=\alpha H\left(X_{1}\right)+\beta H\left(X_{2}\right) .
$$

Proof. We have $H\left(\alpha X_{1}+\beta X_{2}\right)=H\left(\alpha X_{1}\right)+H\left(\beta X_{2}\right)$ as a consequence of Axiom 4 , since scaling does not change comonotonicity. Thus it only remains to show that $H(\gamma X)=\gamma H(X)$ for any non-negative scalar $\gamma$. As a consequence of Axiom 4, $H$ is positively homogeneous in $n \in \mathbb{N}$. Now it is first shown that $H$ is positively homogeneous in $q \in \mathbb{Q}^{+}$. To verify this, note that for arbitrary $m, n \in \mathbb{N}$ one has

$$
\begin{aligned}
& H(X)=H\left(n\left(\frac{1}{n} X\right)\right) \\
\Longleftrightarrow & H(X)=n H\left(\frac{1}{n} X\right) \\
\Longleftrightarrow & \frac{m}{n} H(X)=m H\left(\frac{1}{n} X\right) \\
\Longleftrightarrow & \frac{m}{n} H(X)=H\left(\frac{m}{n} X\right) .
\end{aligned}
$$

Using the fact that the rational numbers lie densely in $\mathbb{R}$, define some non-negative $\gamma:=\lim _{n \rightarrow \infty} q_{n}$, with $q_{n} \in \mathbb{Q}^{+}, \forall n$. Using Axiom 5, one finds that 


$$
\begin{aligned}
H(\gamma X) & =H\left(\lim _{n \rightarrow \infty} q_{n} X\right) \\
& =\lim _{n \rightarrow \infty} H\left(q_{n} X\right) \\
& =\lim _{n \rightarrow \infty} q_{n} H(X) \\
& =\gamma H(X) .
\end{aligned}
$$

Proposition 2. The operator $H$ is convex on the cone of non-negative random prospects.

Proof. Denote $X$ the cone of non-negative random prospects. For $X_{1}, X_{2} \in X$ and arbitrary non-negative scalars $\alpha$ and $\beta$, it follows from Axiom 3 that $H$ is a sublinear functional on $X$, i.e.

$$
H\left(\alpha X_{1}+\beta X_{2}\right) \leq \alpha H\left(X_{1}\right)+\beta H\left(X_{2}\right) .
$$

This is seen as follows. Let $Z_{1}:=\alpha X_{1}, Z_{2}:=\beta X_{2}$, then $Z_{1}, Z_{2}$, and $Z_{1}+Z_{2} \in X$ because $X$ is a cone. Due to Axiom 3 it holds true that

$$
H\left(\alpha X_{1}+\beta X_{2}\right)=H\left(Z_{1}+Z_{2}\right) \leq H\left(Z_{1}\right)+H\left(Z_{2}\right)=\alpha H\left(X_{1}\right)+\beta H\left(X_{2}\right) .
$$

The latter equality is justified by the property of positive homogeneity as verified above. Now to prove that $H$ is convex on $X$, one needs to shown that for some $X_{1}, X_{2} \in X$ and $0 \leq \lambda \leq 1$, it holds true that

$$
H\left(\lambda X_{1}+(1-\lambda) X_{2}\right) \leq \lambda H\left(X_{1}\right)+(1-\lambda) H\left(X_{2}\right) .
$$

Assuming $\alpha+\beta>0$, set $\lambda=\alpha /(\alpha+\beta)$ which concludes the proof.

\section{THE EXPONENTIAL DISTORTION OPERATOR}

As stated in section 1, in a first motivating step for pricing actuaries to use Choquet pricing we propose a particular distortion operator given by a scaled exponential function

$$
\pi(q)=\frac{1-e^{\lambda q}}{1-e^{\lambda}}, 0 \leq q \leq 1, \lambda \geq 0 .
$$

In order to assure convexity, as postulated by $(\pi 2)$, the parameter $\lambda$ is nonnegative. Before a calibration of (9) based on Lorenz order is developed in a second step below, we give some definitions on stochastic order first. 
Definition 1. Two risks $X_{1}$ and $X_{2}$ with positive finite expectation are Lorenz ordered (notation: $X_{1} \leq_{L} X_{2}$ ), if and only if

$$
L_{X_{1}}(p) \geq L_{X_{2}}(p), \forall p \in(0,1) .
$$

With respect to the unit interval the Lorenz function is defined as

$$
L_{X}(p)=\frac{\mathbb{E}\left(F_{X}^{-1}(V) \mid V \leq p\right)}{\mathbb{E}\left(F_{X}^{-1}(V)\right)}, p \in[0,1],
$$

where $V \stackrel{d}{=} U(0,1)$. One can also consider the Lorenz function on the image of $X$ which leads to the expression

$$
L_{X}(x)=\frac{\mathbb{E}(X \mid X \leq x)}{\mathbb{E}(X)}, x \geq 0 .
$$

The latter representation is also denoted the first moment distribution of $X$. For details on Lorenz functions see for instance Johnson, Kotz, and Balakrishnan (1995). Some interesting aspects relating to Lorenz functions and their application in insurance is expounded for instance in Embrechts, Klüppelberg, et al. (1997).

Although stop-loss order (second order stochastic dominance) strikes as being the more well-known concept of stochastic order [as compared to Lorenz order], a definition is given next for completeness [for more details, see e.g. Antal, 1997].

Definition 2. A random prospect $X_{2}$ dominates $X_{1}$ in stop loss order (notation: $X_{1} \leq_{s l} X_{2}$ ), if and only if

$$
\mathbb{E}\left[\left(X_{1}-a\right)^{+}\right] \leq \mathbb{E}\left[\left(X_{2}-a\right)^{+}\right], \forall a \geq 0 .
$$

Well-established results due to the Hardy-Littlewood-Polya Theorem (1929) show that Lorenz order and stop-loss order are equivalent in the presence of equal means [for a detailed discussion see e.g. Shaked, Shantikumar, 1997]. The following theorem and the subsequent reasoning proceed on equal means, with justification given below.

Theorem 1. For any pair of comonotonic and Lorenz ordered random prospects $X_{1}$ and $X_{2}$, with $\mathbb{E}\left(X_{1}\right)=\mathbb{E}\left(X_{2}\right)<\infty$ and $X_{1} \leq_{L} X_{2}$, it holds true that

$$
\mathbb{E}\left(u\left(-X_{1}\right)\right) \geq \mathbb{E}\left(u\left(-X_{2}\right)\right) \Longleftrightarrow H\left(X_{1}\right) \leq H\left(X_{2}\right),
$$

for any increasing concave risk utility function $u$. 
The "if" part in (12) is a consequence of the stop-loss order preserving property of $H$, as shown by Hürlimann (1998). For the "only if" part, the reader is referred to the proof in Niederau [2000, see Appendix E]. The expected utility order [see the lefthand-side in (12)] has been used here to elucidate the concept of quasi risk-neutral pricing [see more comments in the final paragraph of this section]. For a general discussion of expected utility order, including definition, see for instance Varian (1992).

Remark 1. Following the introductory remarks above, due to the assumed equality in means, Theorem 1 can be restated equivalently with respect to stop-loss order.

Remark 2. Equal expectations under $\mathbb{P}$ and Lorenz order $X_{1} \leq_{L} X_{2}$ ensure that the expected utility order on the left-hand side in (12) is well defined for expected utility maximizers with any concave risk utility function $u$. Likewise, note that for any $X \in X, \mathbb{E}(X)=\infty \Rightarrow H(X)=\infty$, in which case a risk $X$ was agreed to be non-insurable in Axiom 1. In the case of the exponential distortion operator one even has $H(X)<\infty$ if and only if $\mathbb{E}(X)<\infty$. The latter equivalence motivates the assumption of finite means to assure that also the inequality on the right-hand side in (12) is well defined.

Remark 3. Theorem 1 may appear restrictive due to the required equality in expectation. But even in presence of random prospects with unequal expected values (the usual case in reality), this apparent limitation can be overcome by using scaled random prospects. In section 2 it has been shown in the proof of Proposition 1 that $H$ is positively homogenous with respect to positive scalars. For scaling, let $\beta \equiv 1 / \mathbb{E}(X)$ to find that $H(X)=\mathbb{E}(X) \cdot H(\beta X)$. Accordingly, with $\hat{X}:=\beta X$, the premium $H(X)$ is determined uniquely by $H(\hat{X})$, provided the actuarial expectation of $X$ exists. Hence, in view of Theorem 1, it is sufficient to concentrate on scaled random prospects for the remaining part of this work.

Remark 4. The result of Theorem 1 can be extended by induction to any set of risky prospects which is totally Lorenz ordered. In the practical part of this paper we will encounter such a set which is characterized by the distribution functions of its elements [cf. Remark 6, section 4.1].

Remark 5. While Theorem 1 is a general result which applies to any distortion operator, a clear-cut rule of calibration with respect to the exponential distortion operator is developed in Niederau [2000, see Lemma 1, section 5.4]. What is shown there is that under Lorenz order the quasi risk-neutral criterion in (12) uniquely relates to a bijective non-linear mapping,

$$
\begin{aligned}
\lambda:[0.5,1) & \rightarrow[0, \infty) \\
\xi & \mapsto \lambda(\xi),
\end{aligned}
$$


which is determined by the implicit equation

$$
\left(e^{\lambda}-\lambda e^{\lambda}-1\right) /\left(\lambda\left(e^{\lambda}-1\right)\right)=\xi .
$$

Here, $\xi$ denotes the point on $[0,1]$ at which the Lorenz function $L_{X}$ associated with some underlying risk $X$ assumes its mean value. Letting $V:=F_{X} \stackrel{d}{=} U(0,1)$, the very same ratio in (14) can be rewritten as

$$
\mathbb{E}\left(V e^{\lambda V}\right) / \mathbb{E}\left(e^{\lambda V}\right) .
$$

Some more interpretation of how the function in (13) relates to risk-aversion, with risk-neutrality being the limit case, is given in Appendix $B$.

To conclude this short review of results, the pricing rule obtained from (14) in essence relates to the Esscher transform as discussed by Bühlmann (1980), according to (15). Here, however, the Esscher transform is applied to the distribution function $F_{X}$ rather than to the random prospect $X$. The difference between these two types of transform is discussed e.g. in Wang (2000).

Up to this point, focus on the exponential distortion operator under the criterion of minimizing relative entropy was not justified. Indeed, Reesor, McLeish, et al. (2003) show that other distortion operators, e.g. normal (socalled 'Wang transform'), beta, piece-wise linear, and others can be motivated under the same criterion. However, it is shown in Appendix B that the exponential distortion operator is the only optimal one under relative entropy minimization, subject to the axioms stated in section 2 and the first moment constraint (14) on the distribution function, such as motivated by Lorenz order. It is for this reason that distortion functions other than the exponential are not considered in this work. Moreover, Reesor, McLeish, et al. (2003) show that a premium derived under the exponential distortion principle is a coherent measure of risk such as discussed in the pertinent literature [see e.g. Dheane, Vanduffel, et al., 2006]. In particular this verifies that the values of Risk Adjusted Capital (RAC) which are derived later in section 4.2 can be interpreted as coherent measure of risk alike.

Note that Theorem 1 holds true for all increasing concave risk utility functions. Therefore, the equivalence in (12) means that all risk-averse (including risk-neutral) market actors order two such risks $X_{1}$ and $X_{2}$ as indicated by $H$. But since in the presence of comonotonic risks $H$ has an expected value representation as in (4), a risk-averse market can be considered quasi risk-neutral with respect to the transformed density $f^{*}(x):=\pi^{\prime}(F(x)) f(x)$. This notion of risk neutrality extends classical expected utility theory, where risk neutrality refers to a market where decision makers order risks uniquely with respect to actuarial expectation, implying linear risk utility functions. In this sense the qualifier "quasi" is used as a sign of caution rather than indicating a concept of risk neutrality beyond the theory of expected utility. 


\section{A PRACTICAL PRICING APPLICATION}

When it comes to practical application, there are many ad hoc methods to calibrate the distortion operator in (9). For instance, one way is to fix a layer premium for some basic layer (e.g. the first million Euro) and calibrate $\lambda$ to match a preset premium for that basic layer. This value of $\lambda$ then determines the risk loading for any higher layer. However, this approach is judgemental in various respects. Even choosing a "meaningful" basic layer and its premium, assumes that the loss dynamics in the basic layer are indicative for the pricing of high-excess layers. The latter approach characterizes layer pricing with increased limit factors (ilf) [see for details Wang, 1995].

By way of contrast, a consistent rule of calibration is developed in section 4.2 below, using Theorem 1 and its extension as alluded to in Remark 4 in section 3. Rather than relying on some layer for calibrating $\lambda$, this rule takes into account the shape of the whole distribution function by means of Lorenz order. In section 4.2, this theory will be applied to an assumed wind risk portfolio such as typically insured by industrial insurers. A member of the Maxwell-Boltzmann, Boese-Einstein, Fermini-Dirac (MBBEFD for short) class of distribution functions as discussed by Bernegger (1997) will be used to model the severity of loss in such a portfolio.

\subsection{Swiss Re exposure curves and Lorenz order}

The MBBEFD function of most practical interest for insurance applications [cf. Bernegger, 1997 for an extensive discussion] is given by

$$
\sigma(x)=\frac{\ln \left(\frac{(g-1) b+(1-g b) b^{x}}{1-b}\right)}{\ln (g b)},
$$

$b>0, b \neq 1, b g \neq 1, g>1, x \in[0,1], \sigma(x) \in[0,1]$, where with $c>0$

$$
\begin{aligned}
& b:=\exp \{3.1-0.15 c(1+c)\}, \\
& g:=\exp \{c(0.78+0.12 c)\} .
\end{aligned}
$$

The underlying distribution function is given by

$$
F_{b, g}(x)=1-\frac{1-b}{(g-1) b^{1-x}+(1-g b)} .
$$

In practical insurance pricing the ensemble (16) and (17) is referred to as the Swiss Re Exposure curve. Subject to the calibration in (17), which will also be 
assumed below, in terms of shape both functions (16) and (18) are completely characterized by the value of $c$. A risk $X$ with distribution function (18) is referred to as being of type sre(c) below. Note that the functions (16) and (18) are not defined for the critical value

$$
\tilde{c}=-0.5+\sqrt{0.25+3.1 / 0.15} \approx 4.07,
$$

where they have a different representation [see Bernegger, 1997, for details].

Some important facts and interpretations are outlined below while again, for the full detail, the reader is referred to Bernegger's work.

1. In both (16) and (18), $x=y / M P L$, where $y$ relates to possible realizations of some underlying random prospect $Y$ which is bounded from above by its Maximum Possible Loss (MPL) which is assumed finite in property insurance. Note that in insurance terminology MPL does not necessarily mean total physical destruction; rather it stands for maximum damage if all risk protection and prevention measures fail. The function in (16) means that an insurance layer in amount of $(x \cdot 100) \%$ of the MPL absorbs $(\sigma(x \cdot 100) \%$ of the total loss expectation. In other words, $\sigma(x)$ is nothing but the limited expected value function of the scaled random variable $X=Y / M P L$. In the present case of property insurance, $Y$ stands for a production plant, a switching station, a warehouse and the like. The calibration given in (17) reproduces certain pricing schemes used in industrial reinsurance markets. In particular, for $c=5$ one obtains the so-called Y 6 curve, a premium allocation scheme used by Lloyd's and many other industrial insurers.

2. If $Y$ is of type sre(c), then

$$
\mathbb{E}(Y)=\frac{M P L_{Y}(g b)(1-b)}{\ln (b)(1-g b)},
$$

the factor

$$
\frac{\ln (g b)(1-b)}{\ln (b)(1-g b)}
$$

just being the expectation of $X=Y / M P L_{Y}$. Unlike the $c$-parameter, the MPL thus serves as a scale parameter.

3. if $Y$ is of type $\operatorname{sre}(c)$, its limited expected value function (LEV) is

$$
L E V_{Y}(y):=\mathbb{E}(Y \mid Y \leq y)=\sigma_{Y}\left(\frac{y}{M P L_{Y}}\right) \mathbb{E}(Y), y \geq 0 .
$$

Proposition 3. Let $Y_{1}$ and $Y_{2}$ be sre $\left(c_{1}\right)$ and sre $\left(c_{2}\right)$, respectively with $c_{1}, c_{2}>0$. Then $Y_{1}$ and $Y_{2}$ are Lorenz ordered. 
Proof. We assume $\mathbb{E}\left(Y_{1}\right)=\mathbb{E}\left(Y_{2}\right)$ which is justified by the scale invariance property of Lorenz order. Recalling the second property above, this equality in expectations implies a parametric constraint given by

$$
\frac{\ln \left(g_{1} b_{1}\right)\left(1-b_{1}\right) \ln \left(b_{2}\right)\left(1-g_{2} b_{2}\right)}{\ln \left(b_{1}\right)\left(1-g_{1} b_{1}\right) \ln \left(g_{2} b_{2}\right)\left(1-b_{2}\right)}=\frac{M P L_{2}}{M P L_{1}}
$$

where the indices 1 and 2 relate to $Y_{1}$ and $Y_{2}$, respectively. Now it needs to be shown that either $Y_{1} \leq_{s l} Y_{2}$, or $Y_{1} \geq_{s l} Y_{2}$. This will already conclude the proof according to Remark 1 in section 3. Assume $c_{1} \leq c_{2}$, then along the contour given by (22) one has

$$
\sigma\left(x_{1} \mid c_{1}\right) \geq \sigma\left(x_{2} \mid c_{2}\right)
$$

with $x_{i}:=y / M P L_{i}, i=1,2$ and $y \geq 0$. But due to the third property above, for all $y \geq 0$ this is equivalent to

$$
\begin{aligned}
& \operatorname{LEV}_{1}(y) \geq \operatorname{LEV}_{2}(y), \\
& \Longleftrightarrow \mathbb{E}\left(Y_{1}\right)-L E V_{1}(y) \leq \mathbb{E}\left(Y_{2}\right)-L E V_{2}(y) \\
& \Longleftrightarrow \mathbb{E}\left(Y_{1} \mid Y_{1} \geq y\right) \quad \leq \mathbb{E}\left(Y_{2} \mid Y_{2} \geq y\right) \\
& \Leftrightarrow Y_{1} \quad \leq_{\mathrm{sl}} Y_{2} \text {. }
\end{aligned}
$$

The latter equivalence is shown for instance by Antal (1997).

Remark 6. The proof highlights that for risks of type sre(c), Lorenz order is determined by the order of the according values of $c$. Moreover, since $c_{1}$ and $c_{2}$ were chosen arbitrarily the set of all risks which are of type sre $(c)$ is even totally ordered under the relation $\leq_{L}$ [see Remark 4, section 3] which motivates the application as outlined in the next section.

\subsection{Calculation of prices and RAC in a quasi risk-neutral setting}

The data shown in table 1 below assume a selection of industrial storm exposures in Belgium and the Netherlands. For the sake of focus, actuarial parameters in this case study, i.e. those relating to the severity and annual frequency of storm losses, are assumed to be given best-estimates and will not be discussed in any detail. The labels, "Loc" and "Ins Val" denote the location and the insured value, respectively. Throughout this section, sums insured, MPL and RAC values are stated in million (mn.) of Euros, while (fair) premium values are stated in thousands of Euros. Due to the typical pathways of European cyclones, locations in Belgium and the Netherlands are usually exposed to the 
TABLE 1

OVERVIEW OF EXPOSURE DATA AND PRICING RESULTS

\begin{tabular}{c|c|r|c|r|r|r|r|r}
\hline \hline Loc & Ins Val & MPL & $c$ & $\mathbb{E}\left(Z_{i}\right)$ & $H\left(Z_{i}\right)$ & RAC & RAC $^{*}$ & RAC $^{+}$ \\
\hline 1 & 261.9 & 26.6 & 4.31 & 21.8 & 49.4 & 1.38 & 21.4 & 25.0 \\
2 & 233.7 & 19.3 & 4.26 & 16.5 & 37.3 & 1.04 & 16.3 & 18.1 \\
3 & 130.2 & 13.2 & 4.17 & 12.4 & 27.8 & 0.77 & 12.0 & 12.4 \\
4 & 64.1 & 8.8 & 4.08 & 9.1 & 20.4 & 0.57 & 8.9 & 8.2 \\
5 & 47.8 & 7.9 & 4.05 & 8.4 & 18.7 & 0.52 & 8.1 & 7.5 \\
6 & 36.7 & 7.3 & 4.04 & 7.9 & 17.6 & 0.49 & 7.7 & 6.9 \\
7 & 29.9 & 6.9 & 4.03 & 7.5 & 16.7 & 0.46 & 7.2 & 6.5 \\
8 & 22.8 & 6.4 & 4.00 & 7.1 & 15.7 & 0.43 & 6.7 & 6.0 \\
9 & 19.3 & 5.7 & 3.96 & 6.6 & 14.7 & 0.41 & 6.4 & 5.4 \\
10 & 14.9 & 4.3 & 3.84 & 5.6 & 12.2 & 0.33 & 5.2 & 4.0 \\
\hline Totals & 861.3 & 106.4 & n.a. & 102.9 & 230.5 & 6.40 & 100 & 100 \\
\hline \hline
\end{tabular}

same storms, causing them to present comonotonic risks. For all locations the value of $c$ differs from the critical value of 4.07, as in (19), indicating that the distribution function in (18) is well defined. Therefore the data satisfy all prerequisites of Theorem 1 and Remark 4, since (besides comonotonicity) these ten locations are completely Lorenz ordered by their value of $c$, according to Proposition 3. A full value cover is assumed for the sake of simplicity, meaning that neither the insured nor the insurer imposes any loss limits other than the location-specific MPL ${ }^{1}$. A common expected loss frequency of 3.5\% percent per location and per annum was assumed. In such a situation theory suggests using a Poisson distribution. However, from a practical viewpoint, in the presence of such a low frequency the probability of more than one loss occurring per year is negligibly low (an argument which is elaborated in some more detail by Daykin, Pentikäinen, et al. (1994) in the context of so-called shadow claims). This is why in this practical study a diatomic distribution with parameters $p_{i}=0.035$ and $n_{i}=1, \forall i=1, \ldots, 10$, respectively, is used for simplicity. The diatomic representation of the loss frequency implies that with probability $p_{i}$ one loss occurs at location $i$ and accordingly, no loss occurs with the counter probability $1-p_{i}$. Thus, treating each location as a single risk unit $X_{i}$, the total portfolio loss can be written as

$$
Z=\sum_{i=1}^{10} Z_{i}=\sum_{i=1}^{10} \mathbb{1}_{\{N=1\}} X_{i},
$$

1 This is clearly an abstraction from reality since insurers would usually sublimit their exposures to naturals perils in order to counteract loss accumulation. 
where

$$
H(Z)=\sum_{i=1}^{10}\left[p_{i} H\left(X_{i}\right)+\left(1-p_{i}\right) H(0)\right]=\sum_{i=1}^{10}\left[p_{i} H\left(\hat{X}_{i}\right) \mathbb{E}\left(X_{i}\right)\right] .
$$

In accordance with the notation introduced before, $\hat{X}_{i}$ refers to the scaled loss variable $X_{i} / E\left(X_{i}\right)$. Here, the pricing functional $H$ takes into account that no loss occurs with probability $1-p_{i}$, which does not give rise to any premium in keeping with Axiom 1. The total expected loss per location is obtained by Wald's identity, i.e. $E\left(Z_{i}\right)=p_{i} E\left(X_{i}\right)$. Recalling (24), $H\left(Z_{i}\right)$ is nothing but $p_{i} H\left(X_{i}\right)$, which is why

$$
H\left(Z_{i}\right) / \mathbb{E}\left(Z_{i}\right)=H\left(X_{i}\right) / \mathbb{E}\left(X_{i}\right) .
$$

The latter ratio can thus be interpreted as the loading factor charged for location $i$ [see table 3 in the Appendix for a set of loading factors as a function of $c$ ].

The determination of the (one-year) Risk Adjusted Capital (RAC), or more precisely, the return on Risk Adjusted Capital (RoRAC), is an important issue to insurers, since (in conjunction with the insurer's $\beta$ from the CAPM) it helps investors to position an insurer relative to their efficient portfolio frontier [see for details e.g. Zweifel, Auckenthaler, 2008]. Now recall that under quasi riskneutrality, the location-specific surcharges $H\left(Z_{i}\right)-E\left(X_{i}\right)$ can be interpreted as certainty-equivalent net cash-flows. Assuming discrete compounding for simplicity, this permits to uniquely determine the respective present value of RAC as

$$
\mathrm{RAC}_{i}=\left[H\left(Z_{i}\right)-\mathbb{E}\left(Z_{i}\right)\right] / r_{f},
$$

where $r_{f}$ denotes the one-year risk-free hurdle rate. The according RAC values are shown in table 1 for an assumed hurdle rate of 2 percent per annum. Total $\mathrm{RAC}$, being defined as

$$
R A C_{t o t}=\sum_{i=1}^{10} R A C_{i}
$$

amounts to about 6 percent of total MPL, or Euro $6.4 \mathrm{mn}$. Table 2 shows that this value is close to the $99.6 \%$ quantile of the aggregate loss distribution of the portfolio risk as given in (23). This implies that only once in 250 years will the aggregate loss burden exceed $R A C_{t o t}$, forcing the insurer to raise capital beyond this value.

It may be worthwhile to emphasize the sensitivity of these estimates to the cutoff point chosen. At the $95 \%$ quantile, no RAC would have been necessary, while at $99 \%$ quantile (a preferred choice in practical applications), RAC would amount to some Euro $3.2 \mathrm{mn}$., just about one-half of the calculated value [see table 2]. More generally, quantile-based rules for the determination of $\mathrm{RAC}$ may expose an insurer to a great potential for error, particularly in the 
TABLE 2

Selected Percentiles of the AGgREgate PORTFOlio distribution

\begin{tabular}{cc}
\hline \hline loss amount & percentile \\
\hline 0 & 96.50 \\
0.71 & 97.00 \\
1.08 & 97.50 \\
1.47 & 98.00 \\
1.98 & 98.50 \\
3.32 & 99.00 \\
5.89 & 99.50 \\
6.45 & 99.60 \\
8.54 & 99.75 \\
\hline \hline
\end{tabular}

presence of highly skewed loss distributions. However, even when using the exact RAC calculation rule, insurers may still be concerned about parameter uncertainty, a topic which is not addressed in this paper but merits mentioning [for details on the treatment of parameter uncertainty see e.g. Wang, 2003]. In table 1, the allocation as derived from the quasi risk-neutral pricing (indicated by $\mathrm{RAC}^{*}$, in percent of $R A C_{t o t}$ ), is juxtaposed to a typical practitioner's rule-ofthumb allocation $R A C^{+}$, in percent of the very same amount $R A C_{t o t}$. The rule of thumb allocates RAC according to the location's relative MPL, such that

$$
\frac{R A C_{i}^{+}}{R A C_{t o t}}=\frac{M P L_{i}}{\sum_{i=1}^{10} M P L_{i}} .
$$

As can be seen from the last column of table 1, this rule biases RAC allocation in favor of the locations with high MPL values. By way of comparison, the allocation based on quasi risk-neutral pricing results in a more balanced RAC allocation. Since RAC is often used not only as a measure of risk tolerance but also as a means to measure underwriting performance, choosing an appropriate allocation rule is of considerable importance. The rule based on quasi risk-neutral pricing can be argued to be preferable because it takes account of the whole distribution function of the risks involved rather than just focussing on the maximum (foreseeable) exposure to loss, a mere reflection of risk aversion. In the present context it recognizes the fact that losses in property insurance do not necessarily occur at locations with large MPL but also hit locations of medium to small size.

\subsection{Discussion}

While providing interesting insights, this case study cannot claim general validity. Usually, insurance portfolios are neither comonotonic nor are they completely 
Lorenz ordered. However, Theorem 1 can be generalized beyond comonotonicity and Lorenz order in the following sense. Denote with $X_{1}$ and $X_{2}$ two single risks (e.g. two locations as in the practical application). Then Axiom 3 implies

$$
H\left(X_{1}+X_{2}\right) \leq H\left(X_{1}\right)+H\left(X_{2}\right)
$$

In turn, provided $X_{1}$ and $X_{2}$ have finite expectation, $H\left(X_{1}\right)$ and $H\left(X_{2}\right)$ are uniquely determined by $H\left(\hat{X}_{1}\right)$ and $H\left(\hat{X}_{2}\right)$. The latter quantities can be uniquely calculated following the logic of Theorem 1 and the calibration motivated in Remark 5, since $X_{1}$ and $X_{2}$ are both Lorenz ordered and perfectly correlated with themselves, hence comonotonic. Proposition 2 [see section 2] ensures that this reasoning also holds true for any convex mixing of $X_{1}$ and $X_{2}$, according to the convexity property of $H$. This may be of relevance for instance in the context of quota-share agreements between primary insurers and reinsurers. In such a case, $X_{1}$ and $X_{2}$ may denote for instance layer risks of the same underlying or even layer risks of different underlyings, e.g. an (excess of loss) layer defined on a property risk blended with an (excess of loss) layer of a liability risk such that the quota-shares on both layers add up to $100 \%$.

These considerations can be used to calculate at least an upper bound RAC for any insurance portfolio. If $P F^{(I)}=\left(Y_{i}\right)_{i=1, \ldots, n}$ denotes some insurance portfolio, $Y_{i}$ single risks for all $i$, and $R A C\left(P F^{(I)}\right)$ the one-year RAC for this portfolio, then the theory predicts

$$
R A C\left(P F^{(I)}\right) \leq \sum_{i=1}^{n}\left[H\left(Y_{i}\right)-\mathbb{E}\left(Y_{i}\right)\right] / r_{f}
$$

This inequality is of interest for at least three reasons. First, from a risk management point of view, it provides a value of the maximum RAC assuming a complete lack of diversification effects in the portfolio under consideration. Second, the ratio

$$
\frac{r_{f} R A C\left(P F^{(I)}\right)}{\sum_{i=1}^{n}\left[H\left(Y_{i}\right)-\mathbb{E}\left(Y_{i}\right)\right]} \leq 1
$$

may serve as an operational measure of diversification effects. Indeed, (27) can be interpreted as the benefit of diversification to potential purchasers of insurance. The closer the ratio on the left-hand side of (27) is to zero the higher the benefit to the insured is. Third, from a shareholder value point of view, excessive RAC, indicated by a violation of (27), is a cause of concern. Too much costly capital would be tied up by underwriting and as a consequence, return to capital is both understated and lower than necessary, to the detriment of shareholders. However, this means that an insurance company runs the risk of 
not being on investors' efficient frontier in terms of expected returns and volatility of returns, unless this shortfall can be made up by a success in capital investments that outperforms the other investors in the capital market [see e.g. Zweifel, Auckenthaler, 2008].

\subsection{Conclusions and outlook}

The objective of this contribution is to derive a quasi risk-neutral pricing rule for insurance that amounts to an expected value, defined under a modified probability measure. While assuring minimum relative entropy with respect to the actuarial probability measure, this modification reflects risk aversion of market actors in the insurance industry. To the extent that insurance risks are comonotonic and exhibit Lorenz order, loading factors such as mirrored in the insurer's Risk Adjusted Capital can be determined uniquely. They call for a higher surcharge to those (scaled) risks dominating under Lorenz order. Moreover, a maximum price can be derived for any portfolio by abstracting from diversification effects, i.e. by pricing every single risk unit independently. Quasi risk-neutral pricing is applied to a set of industrial plants having exposure to storm loss. Location-specific RAC values are calculated and compared to a typical practitioner's rule that allocates RAC according to the location's maximum possible loss (MPL) in proportion to the portfolio's total MPL. Since quasi risk-neutral pricing is based not only on the maximum exposure to loss but also takes into account the shape of the entire loss distribution, it avoids underreserving for risks that have considerable loss potential in the small to medium range which fails to be reflected in their limited MPL values.

In view of further research, the maximum price derived for any portfolio such as motivated in this work may be refined to yield more accurate upper bounds (or even exact values) by including the effects of risk diversification. In the same vein, insurers' capital investments should be taken into account because they provide additional hedging opportunities. These considerations might result in a more general rule for RAC allocation, with RAC determined by a coherent risk measure in the sense of Artzner, Delbaen, et al. (1999). Finally, the relationships between distortion principles, coherent risk measures and the concept of relative entropy merit further exploration, following the suggestion by Reesor, McLeish, et al. (2003).

\section{A. Appendix}

As show in table 3 the sensitivity of the loading factors is highest for values of $c$ up to about 3.5. For higher values of $c$, such as considered in section 4.2, they still increase but at a decreasing rate. These values may appear counterintuitive to insurance practitioners who would usually consider a risk characterized by $c=1$ as being more risky than one with $c=5$ (e.g.) because the former exhibits more exposure to MPL and hence seems to require more risk capital. This apparent paradox can be explained by recalling the separation of 
TABLE 3

LOADING FACTORS AND RELATED PARAMETERS AS A FUNCTION OF THE C-VALUE

\begin{tabular}{cccc}
\hline \hline $\boldsymbol{c}$-value & $\boldsymbol{\xi}$ & $\lambda(\boldsymbol{\xi})$ & loading factor \\
\hline 1.0 & 0.5784 & 0.955 & 1.21 \\
2.0 & 0.6199 & 1.492 & 1.45 \\
2.5 & 0.6335 & 1.676 & 1.62 \\
3.0 & 0.6644 & 2.115 & 1.97 \\
3.5 & 0.6771 & 2.307 & 2.12 \\
4.0 & 0.6856 & 2.439 & 2.22 \\
4.5 & 0.6905 & 2.517 & 2.28 \\
5.0 & 0.6932 & 2.560 & 2.33 \\
5.5 & 0.6946 & 2.583 & 2.37 \\
6.0 & 0.6953 & 2.595 & 2.39 \\
6.5 & 0.6957 & 2.601 & 2.40 \\
7.0 & 0.6960 & 2.606 & 2.41 \\
\hline \hline
\end{tabular}

scale and risk, mentioned in Remark 3 in section 3. According to the parametrization in (14), when $c$ approaches zero this means approaching the deterministic case, i.e. MPL being realized with certainty ${ }^{2}$. While depending on the size of the MPL, such an exposure to loss can call for a great deal of capital. However, in keeping with Axiom 1, this capital will be completely provided by the premium charged, which in this case just coincides with the MPL and therefore with the actuarially fair premium. Hence, in the limit, when $c$ approaches zero, there is no need or even justification (according to the "no rip-off" part of Axiom 1) to charge a premium beyond the actuarially fair value. Conversely, a risk with $c=5$ exhibits quite a skewed loss distribution and the actuarially fair premium is but 1.22 percent of the MPL, regardless of its value ${ }^{3}$. In case of such a risk, the insurer needs to raise extra risk capital in order to fund a potential MPL. The associated opportunity cost of risk capital is reflected in the loading factor of 2.33, as shown in table 3 . A set of comparative loading factors for the generalized Pareto distribution, discussed for instance in Embrechts, Klüppelberg, et al. (1997), can be found in Niederau [2000, see Appendix C].

\section{B. Appendix}

It is mentioned in section 2 that $H$ satisfying the Axioms 1 to 5 is an integral with respect to a distortion function in the sense of (4) while the distortion function is characterized by the properties $(\pi 1)-(\pi 3)$, noted in the very same

\footnotetext{
2 Amunition plants or any industrial plant exposed to vapor cloud explosion may serve as an example.

3 This value can be verified using formula (20).
} 
section. Imposing the implicit equation (14) [see section 3] as equality constraint on the maximizing entropy condition, results in the augmented firstorder condition

$$
\delta\left[-\int_{(a, b)} f(x) \ln f(x) d x+\lambda\left(\int_{(a, b)} x f(x) d x-\xi\right)\right]=0,
$$

which while differentiating with respect to $f$ implies that

$$
\begin{array}{rlrl}
-\int_{(a, b)}\left(\ln f(x) \frac{d}{d x} f(x)+\frac{d}{d x} f(x)-\lambda x \frac{d}{d x} f(x)\right) & d x & =0 \\
\Longrightarrow \ln f(x)+1-\lambda x & & =0 \\
\Longrightarrow \ln f(x) & & =\lambda x-1 \\
\Longrightarrow & f(x) & & =v e^{\lambda x} .
\end{array}
$$

From the necessary prerequisite of $f(x)$ being a proper density function such that $\int_{(0,1)} f(x)=1$, it follows that $\int_{(0,1)} e^{\lambda x}=1 / v$ and hence $v=-\lambda /\left(1-e^{\lambda}\right)$. Inserting the normalizing constant $v$ in the expression of $f(x)$ above, one obtains

$$
f(x)=-\frac{\lambda e^{\lambda x}}{1-e^{\lambda}} .
$$

Integrating with respect to $x$ over the unit interval yields

$$
F(x)=\frac{1-e^{\lambda x}}{1-e^{\lambda}}
$$

which corresponds to the exponential operator of (9) [see section 3], subject to a switch in notation. Hence, the density in (29) is the solution of the problem in (28). However, since the properties $(\pi 1)-(\pi 3)$ have not been specified explicitly as constraints in (28), it needs to be ensured that they are satisfied by (30) to guaranty that the entropy maximizing density found in (29) is still optimal. But $(\pi 1)$ and $(\pi 3)$ are obviously satisfied by (30) because it is just a scaled exponential distribution function, whilst convexity of the very same expression, the requirement in $(\pi 2)$, is verified in Niederau [2000, see Appen$\operatorname{dix} B]$.

While $\lambda$ appears as a Lagrange multiplier in (28), it also has an economic interpretation as alluded to next. The (heuristic) interpretation of a parameter in a distortion function as 'risk-aversion parameter' is not new. Wang (1995) 
for instance alludes to the ph-transform parameter as risk-aversion parameter. In the present case of the exponential distortion, note that in the limit for $\lambda \rightarrow 0$ the distribution function (30) is a uniform distribution scaled to the unit interval. According to (4), in this limit case $H$ just corresponds to the actuarial loss expectation being indicative of risk-neutrality. According to the rule of calibration in (14), the limit case $\lambda=0$ is obtained for degenerate random prospects, defined as being completely characterized by their first moment. In such a case, the coincidence of $H$ with the constant value that such a random prospect assumes for all states of nature implies equality on both sides of (2) in Axiom 1. Some practical intuition in case of the Swiss Re property curves with respect to this limit case (when the characteristic parameter $c$ approaches zero) is already given in the previous Appendix. Indeed, in case of the exponential distortion operator and subject to the assumptions underlying Theorem 1, there is some momentum to the perception of $\lambda$ as a measure of risk-aversion beyond a purely heuristic interpretation. A possible reference for further detail is given in Remark 5 [see section 3].

\section{REFERENCES}

AnTAL, P. (1997) Mathematische Methoden der Rückversicherung, lecture notes ETHZ, Zurich. Artzner, P., Delbaen, F., Eber, J.M. and Heath, D. (1999) Coherent Measures of Risk, Mathematical Finance, 9(3), 203-228.

Bernegger, S. (1997) The Swiss Re Exposure Curves and MBBEFD Distribution Class, Astin Bulletin, 27(1), 99-111.

Bühlmann, H. (1980) An Economic Premium Principle, Astin Bulletin, 11, 52-60.

Daykin, C.D., Pentikäinen, T. and Pesonen, M. (1994) Practical Risk Theory for Actuaries, Monographs on Statistics and Applied Probability, 53, Chapman and Hall.

Delbaen, F. and Haezendonck, J. (1989) A Martingale Approach to Premium Calculation Principles in an Arbitrage-Free Market, Insurance: Mathematics and Economics, 8(4), 269-277.

Denneberg, D. (1994) Non-Additive Measure and Integral, Boston: Kluver Academic Publishers.

Denuit, M., Dhaene, J.S., Goovaerts, M., KaAs, R. and Vyncke, D. (2002a) The Concept of Comonotonicity in Actuarial Science and Finance: Theory, Insurance: Mathematics \& Economics, 31(1), 3-33.

Denuit, M., Dhaene, J.S., Goovaerts, M., KaAs, R. and Vyncke, D. (2002b) The Concept of Comonotonicity in Actuarial Science and Finance: Applications, Insurance: Mathematics \& Economics, 31(2), 133-161.

Denuit, M., Dhaene, J.S., Goovaerts, M. and KaAs, R. (2005) Actuarial Theory for Dependent Risks - Measures, Orders and Models, New York: John Wiley.

Dhaene, J.S., Vanduffel, Q., Tang, M.J., Goovaerts, R., KaAs, R. and Vyncke, D. (2006) Risk Measures and Comonotonicity: A Review, Stochastic Models, 22, 573-606.

Embrechts, P., KlüppelberG, C. and Mikosch, T. (1997) Modelling Extremal Events for Insurance and Finance, Heidelberg: Springer.

Föllmer, H., Schweizer, M. (1991) Hedging of Contingent Claims Under Incomplete Information, Applied Stochastic Analysis (Davis, M. H. and Elliott, R.J., eds.), 389-414, Gordon and Breach.

Gerber, H.U. and Shiu, E.S. (1994) Option Pricing by Esscher Transforms (with discussion), Transactions of the Society of Actuaries, 46, 99-191.

Goovaerts, M.J. and Dhaene, J. (1998) On the Characterization on Wang's Class of Premium Principles, Transactions of the 26th International Congress of Actuaries, 4, 121-134.

Hardy, G., Littlewood, J. and Polya, G. (1929) Some Simple Inequalities Satisfied by Convex Functions, Messenger of Mathematics, 58, 48-152. 
Hürlimann, W. (1998) On Stop-Loss Order and the Distortion Pricing Principle, Astin Bulletin, 28(1), 119-134.

Johnson, N.L., Kotz, S. and Balakrishnan, N. (1995) Continuous Univariate Distributions, New York: John Wiley.

Niederau, H. (2000) Pricing Risk in Incomplete Markets: An Application to Industrial Reinsurance, Doctoral Thesis at the Socioeconomic Institute at the University of Zurich.

Pelsser, A. (2008) On the Applicability of the Wang Transform for Pricing Financial Risks, Astin Bulletin, 38(1), 171-181.

Reesor, R.M. and McLeish, D.L. (2003) Risk, Entropy, and the Transformation of Distributions, North American Actuarial Journal, 7(2), 128-144.

Shaked, M. and Shantikumar, J.G. (1997) Stochastic Orders and their Applications, Boston: Academic Press.

Varian, H.R. (1992) Micro-economic Analysis, New York: W.W. Norton \& Company.

Wang, S., (2003) Cat Bond Pricing using Probability Transforms, Geneva Papers, 278, 19-29.

WANG, S. (2000) A Class of Distortion Operators for Pricing Financial and Insurance Risks, Journal of Risk and Insurance, 67(1), 15-36.

WANG, S. (1995) Insurance Pricing and Increased Limits Ratemaking by Proportional Hazard Transforms, Insurance Mathematics and Economics, 17, 43-54.

Zweifel, P. and Auckenthaler, Ch. (2008) On the Feasibility of Insurer's Investment Policies, Journal of Risk and Insurance, 75(1), 193-206.

\section{HARRY NiEDERAU}

Dr. Niederau Consulting \& Research

Hochstrasse 26

8044 Zurich

Switzerland

E-Mail:info@niederau-research.com

Tel.: +41435370351

Fax: +41435370749

Peter Zweifel

Socioeconomic Institute of the University of Zurich

Hottingerstrasse 10

8032 Zurich

Switzerland

E-Mail:pzweifel@soi.uzh.ch

Tel.: +41446342205

Fax: +41446344907 